\title{
Computing Average Value in Ad Hoc Networks *
}

\author{
Mirosław Kutyłowski ${ }^{1}$ and Daniel Letkiewicz ${ }^{2}$ \\ 1 Inst. of Mathematics, Wrocław University of Technology, \\ mirekk@im.pwr.wroc.pl \\ 2 Inst. of Engineering Cybernetics, Wrocław University of Technology, \\ dletkiew@ict.pwr.wroc.pl
}

\begin{abstract}
We consider a single-hop sensor network with $n=\Theta(N)$ stations using $R$ independent communication channels. Communication between the stations can fail at random or be scrambled by an adversary so that it cannot be distinguished from a random noise.

Assume that each station $S_{i}$ holds an integer value $T_{i}$. The problem that we consider is to replace the values $T_{i}$ by their average (rounded to integer values). A typical situation is that we have a local sensor network that needs to make a decision based on the values read by sensors by computing the average value or some kind of voting.

We design a protocol that solves this problem in $O(N / R \cdot \log N)$ steps. The protocol is robust: a constant random fraction of messages can be lost (by communication channel failure, by action of an adversary or by synchronization problems). Also a constant fraction of stations may go down (or be destroyed by an adversary) without serious consequences for the rest.

The algorithm is well suited for dynamic systems, for which the values $T_{i}$ may change and the protocol once started works forever.
\end{abstract}

Keywords: mobile computing, radio network, sensor network

\section{Introduction}

Ad hoc networks that communicate via radio channels gain importance due to many new application areas: sensor networks used to monitor environment, self-organizing networks of mobile devices, mobile networks used in military and rescue operations.

Ad hoc networks provide many features that are very interesting from practical point of view. They have no global control (which could be either attacked or accidentally destroyed), should work if some stations leave or join the network. So the systems based on ad hoc networks are robust (once they work).

On the other side, it is quite difficult to design efficient algorithms for ad hoc networks. Classical distributed algorithms have been designed for wired environments with quite different communication features. For instance, in many cases one can assume that an hoc networks works synchronously (due to GPS signals); if the network works on a small area, then two stations may communicate directly (single-hop model) and there is

\footnotetext{
* This research was partially supported by Komitet Badań Naukowych grant grant 8T11C 04419.
} 
no communication latency. On the other hand, stations compete for access into a limited number of radio channels. They may disturb each other making the transmission unreadable, if broadcasting at the same time on the same channel. Therefore quite a different algorithmic approach is necessary.

Recently, there have been a lot of research on fundamental issues for ad hoc networks (as a starting point for references see [7]).

Problem Statement. In this paper we consider the following task. Each station $S_{i}$ of a network holds initially an integer value $T_{i}$. The goal is to compute the average value of all numbers $T_{i}$ so that each station changes its $T_{i}$ into the average value. We demand that the numbers held by the stations remain to be integers and their sum is not changed (so at the end, some small differences could be inevitable and the stations hold not exactly the average value but values close to it).

The intuition is that $T_{i}$ might be a physical value measured by a sensor or a preference of $S_{i}$, expressed as an integer value (for instance 0 meaning totally against, 50 meaning undecided and 100 as a fully supporting voice). The network may have to compute the average in order to get an output of a group of sensors or to make a common decision regarding its behavior.

This task, which can trivially be solved for most computing systems (for instance, by collecting data, simple arithmetic and broiadcasting the result) becomes non trivial in ad hoc networks.

Computation Model. We consider networks consisting of identical stations with no ID's (the idea is that it is unpredictable which stations appear in the network and the devices are bulk produced). However, we assume that the stations know $n$, the number of stations in the network, within a constant factor. This parameter is called $N$ for the rest of the paper. (Let us remark that $N$ can be derived by an efficient algorithm [5].)

Communication between stations is accomplished through $R$ independent communication channels labeled by numbers 0 through $R-1$. A station may either send a message through a chosen channel or listen to a chosen channel (but not both at the same time, according to the IEEE 802.11 standard). If more than one station is sending on the same channel, then a collision occurs and the messages on this channel are scrambled. We assume that a station listening to the channel on which there is a collision receives noise and cannot even recognize that a collision has occurred (no-collision-detection model). In this paper we consider only networks that are concentrated in a local area: we assume that if a station sends a message, then every station (except the sender) can hear it. So we talk about single-hop network.

Computation of each station consists of steps. During a step a station may perform a local computation and either send or receive messages through a chosen channel.

For the sake of simplicity of presentation we assume that computation is synchronous. However, our results also hold for asynchronous systems, provided that the stations work with comparable speeds and lack of synchronization may only result in failure of a constant fraction of communication rounds.

We do not use any global clock available to all stations. (In fact, our algorithm can be used to agree upon a common time for other purposes.) 
Design Goals. We design a protocol that has to remain stable and efficient in the following sense:

- Each station may break down or leave the network. However we assume that $\Omega(N)$ stations remain active.

- A message sent by one station is not received by a station that is listening with probability not lower than $p$, where $p<1$ is fixed.

- An adversary, who knows all details about the algorithm, may scramble communication for a constant fraction of the total communication time over all communication channels. (So no "hot spots" of communication pattern of the protocol are admissible - they would be easily attacked by an adversary).

- The protocol has to be suited for dynamic systems, which once started compute the average forever. So it has to be applied in systems where the values $T_{i}$ may change. (For a discussion on dynamic systems see [8].) Preferably, a solution should be periodic with the same code is executed repeatedly.

- The protocol should ensure that the number of steps at which a station transmitting a message or listening is minimized. Also there should be no station that is involved in communication substantially longer than an average station. This is due to the fact that energy consumption is due mainly for radio communication and that battery opereted devices has only limited energy resources.

Former Results. Computing an average value is closely related to load balancing in distributed systems. (In fact, in the later case we have not only to compare the loads but also forward some tasks.) However, the algorithms are designed for wired networks. An exception for it is a solution proposed by Gosh and Muthukrishnan [4]: they propose a simple protocol based on random matchings in the connection graph. In one round of their protocol the load is balanced between the nodes connected by an edge of a matching. This approach is very different from straightforward algorithms that try to gather information and then make decisions based on it. They provide an estimation of convergence of this algorithm to the equal load based on total characteristics of the graph (its degree and the second eigenvalue). Their proof shows decrease of a certain potential describing how much the load is unbalanced.

The protocol of Gosh and Muthukrishnan has been reused for permuting at random in a distributed system [2]. However, in this case the analysis is based on a rapid-mixing property of a corresponding Markov chain and a refined path coupling approach [3].

New Results. We extend the results from [4] and adopt it to the case of a single hop ad hoc network. The issue is that in the protocol of Gosh and Muthukrishnan one needs to show not only that a certain potential decreases fast, but also that there are no "bad points" in the network that have results far from the valid ones (if potential is low, we can only guarantee that the number of bad points is small).

Theorem 1. Consider an ad hoc network consisting of $\Theta(N)$ stations using $R$ communication channels. Let $D$ denote the maximum difference of the form $T_{i}-T_{j}$ at the beginning of the protocol. With high probability, i.e. probability at least $1-\frac{1}{N}$, after executing $O(N / R \cdot(\log N+\log D))$ steps of the protocol: 
- the sum of all values $T_{i}$ remains unchanged and each station keeps one value,

- either all station keep the same value $T_{i}$, or these values differ by at most 1 , or they differ by at most 2 and the number of stations that keep the biggest and the smallest values is bounded by $\varepsilon \cdot N$ for a small constant $\varepsilon$.

\section{Protocol Description}

The protocol repeats a single stage consisting of $3 N / R$ steps, three consecutive steps are called a round. A stage is in fact a step of protocol of Gosh and Muthukrishnan.

Description of a Stage.

1. Each station $S_{i}$ chooses $t, t^{\prime} \in[1, \ldots, N]$ and a bit $b$ uniformly at random (the choices of $t, t^{\prime}$ and $b$ are stochastically independent).

2. Station $S_{i}$ performs the following actions during round $\lceil t / R\rceil$ on the channel $t$ mod $R$.

- If $b=0$, then $S_{i}$ transmits $T_{i}$ at step 1. Otherwise, it transmits $T_{i}$ at step 2.

- At step 3, station $S_{i}$ listens. If a message comes from another station with the $T_{i}$ transmitted and an another value, say $T_{j}$, then $S_{i}$ changes $T_{i}$ as follows:

- if $T_{i}+T_{j}$ is even, then station $S_{i}$ puts $T_{i}:=\frac{1}{2}\left(T_{i}+T_{j}\right)$,

- if $T_{i}+T_{j}$ is odd, then station $S_{i}$ puts $T_{i}:=\left\lfloor\frac{1}{2}\left(T_{i}+T_{j}\right)\right\rfloor$, if its $b$ equals 0 , and $T_{i}:=\left\lceil\frac{1}{2}\left(T_{i}+T_{j}\right)\right\rceil$ otherwise.

3. If $t^{\prime} \neq t$, then during round $\left\lceil t^{\prime} / R\right\rceil$ station $S_{i}$ uses channel $t^{\prime} \bmod R$ :

- it listens during the first two steps,

- it concatenates the messages heard and sends them during the third step.

The idea of the protocol is that $3 N / R$ steps of a single stage are used as a place for $N$ slots in which pairs of stations can balance their values. If everything works fine, then for a given channel at a given round:

- during step 1 a station $S_{u}$ for which $b=0$ sends $T_{u}$,

- during step 2 a station $S_{v}$ for which $b=1$ sends $T_{v}$,

- step 3 is used to avoid Byzantine problems [6]: another station $S_{w}$ repeats $T_{u}$ and $T_{v}$. Otherwise, neither $S_{u}$ nor $S_{v}$ could be sure that its message came through. (An update of only one of the values $S_{u}$ or $S_{v}$ would violate the condition that the sum of all values must not change.)

Of course, such a situation happens only for some slots. However, standard considerations (see for instance [3]) show that the following fact:

Lemma 1. With high probability, during a stage balancing does occur at step 3 for at least $c \cdot N$ slots, where $c$ is a fixed constant, $0<c<1$.

Note that Lemma 1 holds also, if for a constant fraction of slots communication failure occurs or an adversary scrambles messages. Since the stations communicate at randomly chosen moments, it is difficult for an adversary to attack only some group of stations. 


\section{Analysis of the Protocol}

The analysis consists of three different phases (even if the stations behave in exactly the same way all the time). In Phase I we show that some potential function reaches a certain low level - this part is borrowed from [4]. In Phase II we guarantee with high probability that all stations deviate by at most $\beta$ from the average value. Then Phase 3 is used to show that with high probability all stations hold one of at most 3 consecutive values.

In order to simplify the presentation we call the stations $S_{1}, \ldots S_{n}$, even if the algorithm does not use any ID's of the stations.

\subsection{Phase I}

Let $T_{t, j}$ denote the value of $T_{j}$ hold by $S_{j}$ immediately after executing stage $t$. Let $T$ denote the average value, that is, $T=\frac{1}{n} \sum_{i=1}^{n} T_{0, i}$.

We examine the values $x_{t, j}=T_{t, j}-T$. In order to examine the differences from the average value we consider the following potential function:

$$
\Delta_{t}=\sum_{i=1}^{n} x_{t, i}^{2}
$$

Claim A. $\mathbf{E}\left[\Delta_{t+1}\right] \leq \rho \cdot \Delta_{t}+\frac{n}{4} \quad$ for some constant $\rho<1$.

Proof. We first assume that new values hold by two stations after balancing their values become equal (possibly reaching non-integer values). Then we make an adjustment to the real situation when the values must remain to be integers.

By linearity of expectation,

$$
\mathbf{E}\left[\Delta_{t+1}\right]=\mathbf{E}\left[\sum_{i=1}^{n} x_{t+1, i}^{2}\right]=\sum_{i=1}^{n} \mathbf{E}\left[x_{t+1, i}^{2}\right]
$$

So now we inspect a single $\mathbf{E}\left[x_{t+1, i}^{2}\right]$. As already mentioned, with a constant probability $\delta$ station $S_{i}$ balances $T_{t, j}$ with some other station, say with $S_{s}$. Assume that the values held by $S_{i}$ and $S_{s}$ become equal. So $x_{t+1, i}$ and $x_{t+1, s}$ become equal to $z=\frac{1}{2}\left(x_{t, i}+x_{t, s}\right)$. Therefore

$$
\begin{aligned}
\mathbf{E}\left[x_{t+1, i}^{2}\right] & =(1-\delta) \cdot x_{t, i}^{2}+\delta \cdot \mathbf{E}\left[\left(\frac{x_{t, i}+x_{t, s}}{2}\right)^{2}\right] \\
& =(1-\delta) \cdot x_{t, i}^{2}+\delta \cdot \mathbf{E}\left[\frac{1}{4} x_{t, i}^{2}+\frac{1}{4} x_{t, s}^{2}+\frac{1}{2} x_{t, i} \cdot x_{t, s}\right] \\
& =\left(1-\frac{3}{4} \delta\right) \cdot x_{t, i}^{2}+\frac{1}{4} \delta \cdot \mathbf{E}\left[x_{t, s}^{2}\right]+\frac{1}{2} \delta \cdot \mathbf{E}\left[x_{t, i} \cdot x_{t, s}\right]
\end{aligned}
$$

Since $s$ is uniformly distributed over $\{1, \ldots, n\}$, we get

$$
\mathbf{E}\left[x_{t, s}^{2}\right]=\sum_{j=1}^{n} \frac{1}{n} \cdot x_{t, j}^{2}=\frac{1}{n} \cdot \Delta_{t} .
$$


The next expression we have to evaluate is

$$
\mathbf{E}\left[x_{t, i} \cdot x_{t, s}\right]=\sum_{j=1}^{n} \frac{1}{n} \cdot\left(x_{t, i} \cdot x_{t, j}\right)=\frac{1}{n} \cdot x_{t, i} \cdot \sum_{j=1}^{n} x_{t, j} .
$$

Obviously, $\sum_{j=1}^{n} x_{t, j}=0$. So $\mathbf{E}\left[x_{t, i} \cdot x_{t, s}\right]$ equals 0 and finally,

$$
\mathbf{E}\left[x_{t+1, i}^{2}\right]=\left(1-\frac{3}{4} \delta\right) \cdot x_{t, i}^{2}+\frac{1}{4} \delta \cdot \frac{1}{n} \cdot \Delta_{t} .
$$

When we sum up all expectations $\mathbf{E}\left[x_{t+1, i}^{2}\right]$ we get

$$
\begin{aligned}
\mathbf{E}\left[\Delta_{t+1}\right] & =\sum_{i=1}^{n}\left(\left(1-\frac{3}{4} \delta\right) \cdot x_{t, i}^{2}+\frac{1}{4} \delta \cdot \frac{1}{n} \Delta_{t}\right) \\
& =\left(1-\frac{3}{4} \delta\right) \cdot \Delta_{t}+\frac{1}{4} \delta \cdot \Delta_{t}=\left(1-\frac{1}{2} \delta\right) \cdot \Delta_{t} .
\end{aligned}
$$

Now, let us consider the case when $T_{t, i}+T_{t, s}$ (or equivalently $x_{t, i}+x_{t, s}$ ) is odd. Let us see how it contributes to the change of $\Delta_{t+1}$ compared to the value computed previously. In the simplified case, $S_{i}$ and $S_{s}$ contribute

$$
2\left(\frac{x_{t, i}+x_{t, s}}{2}\right)^{2}
$$

to the value of $\Delta_{t+1}$. Now, this contribution could be

$$
\left(\frac{x_{t, i}+x_{t, s}+1}{2}\right)^{2}+\left(\frac{x_{t, i}+x_{t, s}-1}{2}\right)^{2} \text {. }
$$

For every $y$,

$$
\left(\frac{y+1}{2}\right)^{2}+\left(\frac{y-1}{2}\right)^{2}=2 \cdot\left(\frac{y}{2}\right)^{2}+\frac{1}{2}
$$

so we have to increase the value computed for $\Delta_{t+1}$ by at most $\frac{1}{2}$ for each pair that has established a link. It follows finally that

$$
\mathbf{E}\left[\Delta_{t+1}\right] \leq \rho \cdot \Delta_{t}+\frac{n}{4}
$$

for $\rho=\left(1-\frac{1}{2} \delta\right)$.

Claim B. After $\tau_{0}=O(\log D+\log n)$ stages, $\Delta_{\tau_{0}} \leq \alpha \cdot n+1$ with probability $1-O\left(\frac{1}{n^{2}}\right)$.

Proof. Let $\nabla_{t}=\Delta_{t}-\alpha n$, for $\alpha=\frac{1}{4(1-\rho)}$. By inequality (1)

$$
\begin{array}{r}
\mathbf{E}\left[\nabla_{t+1}\right]=\mathbf{E}\left[\Delta_{t+1}-\alpha n\right] \leq \rho \cdot \Delta_{t}+\frac{n}{4}-\alpha n= \\
\rho \cdot \nabla_{t}+\rho \alpha n+\frac{n}{4}-\alpha n=\rho \cdot \nabla_{t} .
\end{array}
$$

It follows that $\mathbf{E}\left[\nabla_{t+1}\right] \leq \rho \cdot \mathbf{E}\left[\nabla_{t}\right]$. Let $\tau_{0}=\log _{\rho^{-1}} D \cdot n^{2}$. Then $\mathbf{E}\left[\nabla_{\tau_{0}}\right] \leq n^{-2}$. So by Markov inequality, $\operatorname{Pr}\left[\nabla_{\tau_{0}} \geq 1\right] \leq n^{-2}$. We conclude that $\operatorname{Pr}\left[\Delta_{\tau_{0}}<1+\alpha n\right]$ is at least $1-n^{-2}$. 


\subsection{Phase II}

We assume that $\Delta_{\tau_{0}}<1+\alpha n$. Let $\beta=2 \sqrt{\alpha}$. Let $B=B_{t}$ be the set of stations $S_{i}$ such that $\left|x_{t, i}\right|>\beta$, and $G=G_{t}$ be the set of stations $S_{j}$ such that $\left|x_{t, j}\right|=\beta$.

Claim C. $|B \cup G|<\frac{1}{4} n+O(1) \quad$ for each $t \geq \tau_{0}$.

Proof. The stations from $B \cup G$ contribute at least $|B \cup G| \cdot \beta^{2}=|B \cup G| \cdot 4 \alpha$ to $\Delta_{t}$. Since $\Delta_{t} \leq \Delta_{\tau_{0}}<\alpha n+1$, we must have $|B \cup G|<\frac{1}{4} n+\frac{1}{4 \alpha}$.

Now we define a potential function $\Delta^{\prime}$ used to measure the size of $B_{t}$.

Definition 1. For station $S_{i} \in B_{t}$ define $\tilde{x}_{i, t}=x_{i, t}-\beta$ and as a zero otherwise. Then

$$
\Delta_{t}^{\prime}=\sum_{i \in B_{t}} \tilde{x}_{i, t}^{2}
$$

Claim D. $\mathbf{E}\left[\Delta_{t+1}^{\prime}\right] \leq \mu \cdot \Delta_{t}^{\prime} \quad$ for some constant $\mu<1$.

Proof. We consider a single station $S_{i} \in B_{t}$, with $x_{i, t}>\beta$. By Claim C, with a constant probability it communicates with a station $S_{j} \notin B_{t} \cup G_{t}$. In this case, station $S_{j}$ may join $B$, but let us consider contribution of stations $S_{i}$ and $S_{j}$ to $\Delta_{t+1}^{\prime}$. Let $\delta=\beta-x_{j, t}$. Then:

$$
\tilde{x}_{i, t+1}^{2}+\tilde{x}_{j, t+1}^{2} \leq 2 \cdot\left\lceil\frac{\tilde{x}_{i, t}-\delta}{2}\right\rceil^{2}<2 \cdot\left\lceil\frac{\tilde{x}_{i, t}-1}{2}\right\rceil^{2} \leq 2 \cdot\left(\frac{\tilde{x}_{i, t}}{2}\right)^{2}=\frac{\tilde{x}_{i, t}^{2}}{2} .
$$

If $S_{i}$ communicates with a station $S_{j} \in B_{t} \cup G_{t}$, then obviously

$$
\tilde{x}_{i, t+1}^{2}+\tilde{x}_{j, t+1}^{2} \leq \tilde{x}_{i, t}^{2}+\tilde{x}_{j, t}^{2} .
$$

We may apply a simple bookkeeping technique assigning the contribution of stations to $\Delta_{t+1}^{\prime}$ so that the expected value of contribution of $S_{i}$ is bounded by $\mu \cdot \tilde{x}_{i, t}^{2}$ for some constant $\mu$ (we assign to $S_{i}$ the contribution of $S_{i}$ and $S_{j}$ in the first case, and contribution of $S_{i}$ in the second case). Since by linearity of expectation $\mathbf{E}\left[\Delta_{t+1}^{\prime}\right]$ equals the sum of expected values of these contributions, $\mathbf{E}\left[\Delta_{t+1}^{\prime}\right] \leq \mu \cdot \mathbf{E}\left[\Delta_{t}^{\prime}\right]$.

Claim E. For $t \geq \tau_{0}+T$, where $T=O(\log n)$, the set $B_{t}$ is empty with probability $1-O\left(\frac{1}{n^{2}}\right)$.

Proof. By Claim D, E $\left[\Delta_{\tau_{0}+t}^{\prime}\right] \leq \mu^{t} \cdot \mathbf{E}\left[\Delta_{\tau_{0}}^{\prime}\right]$. On the other hand, $\mathbf{E}\left[\Delta_{\tau_{0}}^{\prime}\right] \leq \mathbf{E}\left[\Delta_{\tau_{0}}\right]<$ $\alpha \cdot n+1$. Let $T=\log _{\mu^{-1}}\left(2 \alpha \cdot n^{3}\right)$ and $t \geq T$. Then $\mathbf{E}\left[\Delta_{\tau_{0}+t}^{\prime}\right] \leq n^{-2}$. So by Markov inequality, $\operatorname{Pr}\left[\Delta_{\tau_{0}+t}^{\prime} \geq 1\right] \leq n^{-2}$. Observe that $\Delta^{\prime}$ takes only integer values, so $B_{\tau_{0}+t}$ is empty, if $\Delta_{\tau_{0}+t}^{\prime}<1$. 


\subsection{Phase III}

Let $\tau_{1}=\tau_{0}+T$, where $T$ satisfies Claim E. Then for $t>\tau_{1}$ we may assume that for no station $\left|x_{i, t}\right| \geq \beta$. Our goal now is to reduce the maximal value of $\left|x_{i, t}\right|$. We achieve this in at most $2 \beta-1$ subphases, each consisting of $O(\log n)$ stages: during each subphase we "cut off" one of the values that can be taken by $x_{i, t}$. Always it is the smallest or the biggest value.

Let $V(s)=V_{t}(s)$ be the set of stations, for which $x_{i, t}$ takes the value $s$. Consider $t_{1}>\tau_{1}$. Let $l=\min \left\{x_{i, t_{1}}: i \leq n\right\}$ and and $g=\max \left\{x_{i, t_{1}}: i \leq n\right\}$.

Assume that $l+1<g-1$ (so, there are at least four values of numbers $x_{i, t_{1}}$ ). We show that for $t=t_{1}+O(\log n)$ either $V_{t}(l)=\emptyset$ or $V_{t}(g)=\emptyset$.

Obviously, no station may join $V_{t}(l)$ or $V_{t}(g)$, so their sizes are non-increasing. Now consider a single stage. Observe that $\left|V_{t}(l) \cup V_{t}(l+1)\right| \leq \frac{1}{2} n$ or $\left|V_{t}(g) \cup V_{t}(g-1)\right| \leq \frac{1}{2} n$. W.1.o.g. we may assume that $\left|V_{t}(g) \cup V_{t}(g-1)\right| \leq \frac{1}{2} n$. Consider a station $S_{i} \in V_{t}(g)$. With a constant probability $S_{i}$ communicates with a station $S_{j}$ that does not belong to $V_{t}(g) \cup V_{t}(g-1)$. Then station $S_{i}$ leaves $V_{t}(g)$ and $S_{j}$ remains outside $V_{t}(g)$. Indeed, the values $x_{i, t}$ and $x_{j, t}$ differ by at most 2 , so $x_{i, t+1}, x_{j, t+1} \leq x_{i, t}-1$. It follows that $\mathbf{E}\left[\left|V_{t+1}(l)\right|\right] \leq \psi \cdot\left|V_{t}(l)\right|$ for some $\psi<1$.

We see that in a single stage we expect either $\left|V_{t}(l)\right|$ or $\left|V_{t}(g)\right|$ to shrink by a constant factor. Using Markov inequality as in the proof of Claim E we may then easily derive the following property:

Claim F. For some $T^{\prime}=O(\log n)$, if $t>T^{\prime}$, then with probability $1-O\left(\frac{1}{n^{2}}\right)$ either the set $V_{\tau_{1}+t}(l)$ or the set $V_{\tau_{1}+t}(g)$ is empty.

By Claim F, after $O(\beta \log n)=O(\log n)$ stages we end up in the situation in which there are at most three values taken by $x_{i, t}$. Even then, we may proceed in the same way as before in order to reduce the sizes of $V_{t}(l)$ or $V_{t}(g)$ as long as one of these sets has size $\Omega(n)$. So we can derive the following claim which concludes the proof of Theorem 1:

Claim G. For some $T^{\prime \prime}=O(\log n)$, for $\tau_{2}=\tau_{1}+T^{\prime \prime}$ and $t \geq \tau_{2}$ with probability $1-O\left(\frac{1}{n^{2}}\right)$ either $x_{i, t}$ takes only two values, or there are three values and the number of stations holding the smallest and the largest values is at most $\gamma \cdot n$.

\section{Properties of the Protocol and Discussion}

Changes in the Network. By a simple examination of the proof we get the following additional properties:

- the result holds even if a constant fraction of messages is lost. This only increases the number of stages by a constant factor.

- if some number of stations goes down during the execution of the protocol, then the final values do not concentrate around the average value of the original values of the stations that have survived, but anyway they differ by at most 2 . If a new station joins the network and its value deviates from the values hold by the rest of the stations, then we may proceed with the same analysis. Conclusions regarding 
the rate of convergence and the rate at which new stations emerge can be derived as before.

Energy Efficiency. Time complexity is not the most important complexity measure for mobile networks. Since the devices are powered by batteries, it is important to design algorithms that are energy efficient (otherwise, the network may fail due to exhaustion of batteries). The main usage of energy is for transmitting messages and listening to the communication channel. Energy usage of internal computations and sensors is substantially smaller and can be neglected. Surprisingly, for transmitting and listening comparable amounts of energy are necessary.

A properly designed algorithm should require a small number of messages (not only messages sent, but also messages awaited by the stations). Additionally, the differences between the number of messages sent or awaited by different stations should be as small as possible. The reason is that with similar energy resources no station should be at higher risk of going down due to energy exhaustion.

In out algorithm energy usage of each station is $\Theta(\log n)$. This is optimal since we need that many sending trials to ensure that its value has been transmitted successfully with high probability in the presence of constant probability of transmission failures.

Protocol Extensions - Getting Exactly one Value. Our algorithm leaves the network in a state in which there might be 3 different values. The bound from Theorem 1 regarding behavior of the algorithm cannot be improved. Indeed, consider the following example: assume that initially exactly one station holds value $T-1$, exactly one station holds $T+1$, and the rest has value $T$. Then in order to get into the state when all stations get value $T$ we need that the station with $T-1$ communicates with the station with value $T+1$. However, probability that it happens during a single stage is $\Theta(1 / N)$. Therefore, probability that these two station encounter each other within logarithmic number of stages is $O(\log N / N)$.

Once we are left with the values that differ from the average value by less than two, it is quite reasonable to start a procedure of computing the minimum over all active stations. In fact, it suffices to redefine part 3 of a stage: instead of computing the average of two values both stations are assigned the smaller of their two values. Arguing as in Claim E, we may easily show that after $O(\log n)$ stages with high probability all stations know the minimum.

If each station knows the number $n$ of the active stations, a simple trick may be applied to compute the average value exactly. At the beginning, each value is multiplied by $n$. Then the average value becomes $s=\sum T_{i}$ and it is an integer. So after executing the protocol we end up in the second situation described in Theorem 1 or all stations hold the same value. In order to get rid of the first situation a simple protocol may broadcast the minimal and maximal values to all stations within $O(\log N)$ steps. Then all stations may find $s$ and thereby the average $s / n$.

Dynamic Processes. For a dynamic process, in which the the values considered are changing (think for instance about the output of sensors), we may observe that the protocol works quite well. For the sake of discussion assume that the values may only increase. If we wish to ignore the effect of increments of the values we may think about 
old units of the values existing at the beginning of the protocol, and the new ones due to incrementing the values. When executing part 3 of a stage and "allocating" the units to stations $A$ and $B$ we may assume that first the same (up to 1) amount of old units is given to $A$ and $B$ and afterwards the new units are assigned. In this way, the new units do not influence the behavior of the old ones. So a good distribution of the old units will be achieved as stated by Theorem 1 despite the fact that the values have changed.

Security Issues. Since the stations exchange information at random moments an adversary which only disturbs communication can only slow down the rate of convergence. However, if it knows that there is a station with a value $X$ that differs a lot from the average, it may increase its chances a little bit: if $X$ has not occurred so far during a stage, then it might be advantageous for the adversary to scramble the rest of the stage making sure that $X$ remains untouched. Of course, serious problems occur when an adversary can fake messages of the legitimate stations.

If the legitimate stations have a common secret, say $K$, then the problems can be avoided. Faking messages becomes hard, when the messages are secured with MAC code using $K$. In order to avoid the first problem it is necessary to encipher all messages (together with random nounces). In this case an adversary cannot say which values are exchanged by the algorithm. The only information that might be derived is the fact that somebody has transmitted at a given time. But this seems not to bring any substantial advantage, except that then it could be advantageous to attack the third step of a round. Encryption with a symmetric algorithm should be no problem regarding speed differences between transmission and internal computations.

\section{Acknowledgment}

We thank Artur Czumaj for some ideas developed together and contained in this paper.

\section{References}

1. Chlebus, B. S.: Randomized communication in radio networks. A chapter in „Handbook on Randomized Computing” (P. M. Pardalos, S. Rajasekaran, J. H. Reif, J. D. P. Rolim, Eds.), Kluwer Academic Publishers, 2001, vol. I, 401-456

2. Czumaj, A., Kanarek, P., Kutyłowski, M., and Loryś, K.: Distributed stochastic processes for generating random permutations. ACM-SIAM SODA'99, 271-280

3. Czumaj, A., Kutyłowski, M.: Generating random permutations and delayed path coupling method for mixing time of Markov chains. Random Structures and Algorithms 17 (2000), 238-259

4. Gosh, B., Muthukrishnan, S.: Dynamic Load Balancing in Parallel and Distributed Networks by Random Matchings. JCSS 53(3) (1996), 357-370

5. Jurdziński, T., Kutyłowski, M., Zatopiański, J.: Energy-Efficient Size Approximation for Radio Networks with no Collision Detection. COCOON'2002, LNCS 2387, Springer-Verlag, 279-289

6. L. Lamport, R. Shostak and M. Pease: The Byzantine Generals Problem. ACM TOPLAS 4 (1982), 382-401

7. I. Stojmenovič (Ed.): Handbook of Wireless Networks and Mobile Computing, Wiley, 2002

8. E. Upfal: Design and Analysis of Dynamic Processes: A Stochastic Approach, ESA'1998, LNCS 1461, Springer-Verlag, 26-34 